This copy is authors' original pre-print version and may slightly differ from the official published version.

Full citation:

Antila, J., Karhu, T., Mottonen, M., Harkonen, J. and Belt, P. (2008) 'Reducing test costs in electronics mass-production', International Journal of Services and Standards, Vol. 4, No. 4, pp. 393-406.

DOI: http://dx.doi.org/10.1504/IJSS.2008.020055

\title{
Reducing test costs in electronics mass-production
}

\section{Jukka Antila and Timo Karhu}

Nokia Siemens Networks, P.O. Box 319,

FI-90651 Oulu, Finland

E-mail: jukka.antila@nsn.com

E-mail: timo.karhu@nsn.com

\section{Matti Mottonen*, Janne Harkonen and Pekka Belt}

Department of Industrial Engineering and Management, University of Oulu, Finland, P.O. Box 4610,

FI-90014 University of Oulu, Finland

E-mail: matti.mottonen@oulu.fi

E-mail: janne.harkonen@oulu.fi

E-mail: pekka.belt@oulu.fi

*Corresponding author

\begin{abstract}
This paper studies the simultaneous optimisation of quality and costs in mass-production of complex electronics products. Testing has become a critical bottleneck for assuring quality, requiring a large amount of time, and resources. The volume of especially functional testing must be minimised to reduce costs. Sampling is a potential way to obtain this. Unfortunately, existing sampling methods are not functional in the modern electronics environment with multiple tests. This paper presents new efficient methods, based on continuous sampling plan (CSP) procedures. The applicability of the developed methods is confirmed empirically by analysing and simulating real industrial data.
\end{abstract}

Keywords: Testing; Simulation; Standards; Sampling; CSP; Electronics; Manufacturing; Services. 


\section{Introduction}

Modern electronics products with numerous features are getting increasingly complex, while the quality requirements are tighter than ever. Testing is required to ensure the functionality of these features. The volume of testing is vast, and is responsible for a major share of development and production costs in the modern high tech industry (e.g. Ryu et al., 2006). The production volumes have constantly grown in telecom, as well as in many other sectors. At the same time, tougher global competition forces companies simultaneously to reduce costs and to shorten the development times and production leadtimes (e.g. Helo, 2004).

Production testing can be roughly divided into process and functional testing. Process testing evaluates the production process itself, rather than the functioning of products. For cost reasons, the share of process testing should generally be maximised and that of functional testing minimised (e.g. Kerkhoff, 2005). Functional testing, however, is unavoidable, and typically is necessary for ensuring faultless operation of the product and for meeting product specifications. Especially, in industries where quality and reliability requirements are of great importance (e.g., telecom, the medical field, and avionics) functional testing can be quite an extensive and time-consuming operation.

Even though the automation rate is high in functional testing, it is often a bottleneck (e.g. Guo et al., 2007; Belt et al., 2008), and also a major source of costs in modern electronics production. In many cases, production testing for a new product starts with a large set of tests. Later, various methods are used to reduce the number of these tests (e.g. Gershon and Christobek, 2006). However, tens or even hundreds of functional tests are run on each manufactured product with expensive high-accuracy instruments.

A traditional way for decreasing inspection costs is sampling of tests. Conventional 'lot-based' sampling methods are, however, not practical for continuous production. Continuous sampling plans (CSPs) have been developed to address this issue. CSPs are a group of sampling methods suitable for an environment where short lead times are essential, and inventories are to be avoided.

Even though CSP methods are excellent tools for continuous production, they have limitations in a multi-test environment typical for modern electronics production. This is why sampling is not widely used for functional testing in the electronics industry.

This study concentrates on analysing methods for developing the utilisation of functional testing more efficient and effective, without sacrificing the quality seen by the end-user. The study clarifies how CSP methods could be better implemented in functional testing, in modern high tech production, where a vast number of tests are conducted.

The above mentioned can be condensed into the research question:

RQ. What type of sampling method suits continuous electronics mass-production with numerous tests?

The research question is addressed both, theoretically through literature, and empirically by analysing and simulating real industrial data. 


\section{Theory}

Sampling is a potential approach for optimising quality cost ratio in electronics mass production. Product quality seen by the end-user cannot be sacrificed, when considering ways to reduce functional tests in production. Sampling methods, for reducing testing, are interesting as they allow knowing the quality risk levels (e.g. Vasudevan and Selladurai, 2006). Therefore, the accepted risk level, for customers receiving a faulty product, must be defined, prior to applying sampling techniques. The best possible assurance for product functionalities can only be obtained via $100 \%$ test coverage, which, however, is not economically viable (e.g. Chen, 2005).

The quality risk of continuous sampling plan (CSP) methods is defined through the average outgoing quality limit (AOQL). AOQL is the worst possible quality passing through a sampling plan (e.g. Groomer and Murthy, 2003). Other significant parameters, for understanding CSPs, include $i$ and $f$. The parameter $i$, also known as clearing interval, defines the number of products needed to be tested fully (100\%) before sampling can be started. The $f$ is the fraction of units to be tested, after the sampling has been triggered. For example, $f=1 / 10$ means that every tenth product is tested. The main parameters of CSPs (AOQL, $i$, and $f$ ) are interdependent, and can be defined by using diagrams, tables, or equations (e.g. Chen, C-H. 2004; Montgomery, 2004; Dodge, 1943; Juran, 1988; Stephens, 2001; MIL-STD-1235C, 1974; Groomer and Murthy, 2003). Several combinations of $i$ and $f$ can result in the same AOQL. After defining two of these three parameters, the third one can be calculated.

\subsection{Conventional Continuous Sampling Plans}

CSPs are significant methods for reducing tests in production, and thus have been studied for a relatively long period of time. Already, Dodge (1943) introduced the first continuous sampling plan CSP-1. Almost a decade later Dodge and Torrey (1951) developed the CSP-1 plan further and presented two additional plans CSP-2 and CSP-3. Since, several authors have investigated and modified these sampling plans further (see for example, Chen and Chou, 2004; Balamurali and Jun, 2006; Chen and Chou, 2002; Derman et al., 1957; Balamurali and Subramani 2004; Govindaraju, 2000; Lieberman and Solomon, 1955). Some of these plans have also been adopted as US military standards (e.g. MIL-STD-1235C, 1974). Nevertheless, CSP-1 is the simplest and probably the most used continuous sampling plan (Balamurali et al., 2005).

For simplicity, this study focuses on three plans: CSP-1, CSP-2, and CSP-3. All of these are based on a procedure where, first, all products are tested until a set clearing interval $(i)$. The clearing interval requires that no defects are found when inspecting 100 $\%$. After the test qualifies for the set interval $(i)$, a fraction $(f)$ of the units are tested. Once a defective product is identified in sampling, testing returns to the $100 \%$ level, or certain additional control measures commence, depending on the used plan. Should testing return to $100 \%$, a new clearing interval is required to start sampling again.

Figure 1 illustrates the procedures for the discussed three continuous sampling plans. The procedure for CSP-1 is as follows: Use $100 \%$ inspection at the start. When $i$ successive units are found acceptable, full testing is replaced with sampling fraction $f$. Sampling continues until a defective unit is found, and the procedure is restarted (e.g. Juran, 1988; Dodge, 1943).

CSP-2 is often preferred over CSP-1 as the return to $100 \%$ testing does not occur immediately upon detection of a defective unit. An additional parameter, $k$, is introduced 
in CSP-2. Once a defective unit is found, $k$ successive samples are tested at $100 \%$ to find additional assurance. Should this alert period be completed without further defects, sampling continues. However, if a defect is found in $k$ successive samples, the entire procedure is restarted. Nevertheless, compared to CSP-1, the CSP-2 has a downside of requiring a lengthened clearing interval $i$ in order to reach the same AOQL with the same fraction $f$. (e.g. Juran, 1988; Dodge and Torrey, 1951).

The CSP-3 introduces an additional refinement to the previous plans. This is done with the purpose of providing extra protection against the variation of quality. Once the first defective unit is detected, the next four units are inspected. Should another defect be found among these four units, testing returns to the $100 \%$. However, if all four units are defect free, the plan continues as in CSP-2, and the next $k$ samples are controlled. (e.g. Juran, 1988; Dodge and Torrey, 1951). 
Figure 1. Procedures for three discussed CSPs

CSP-1

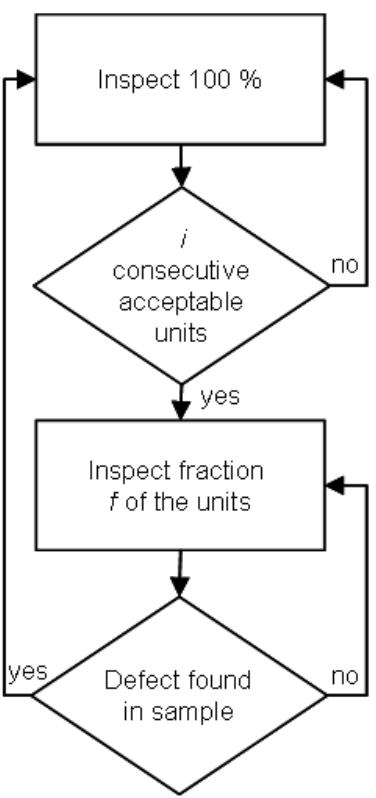

CSP-2

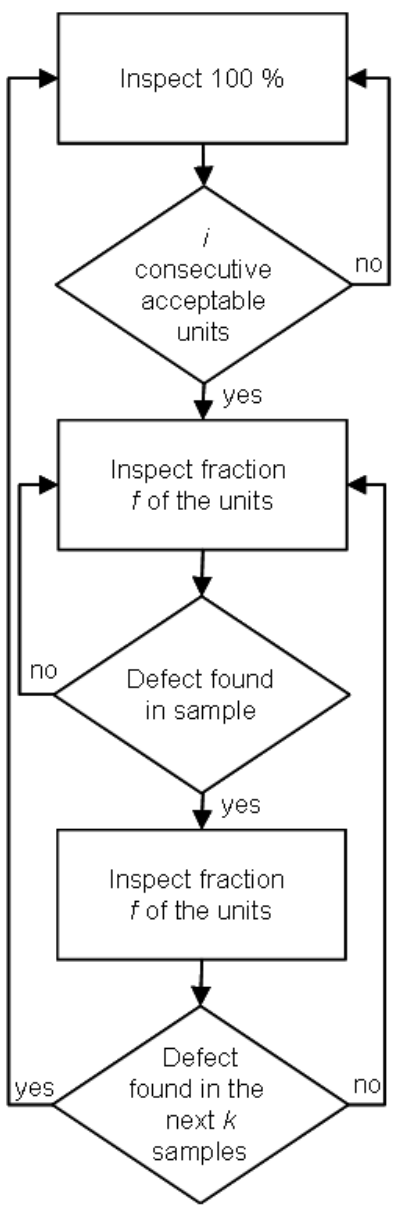

CSP-3

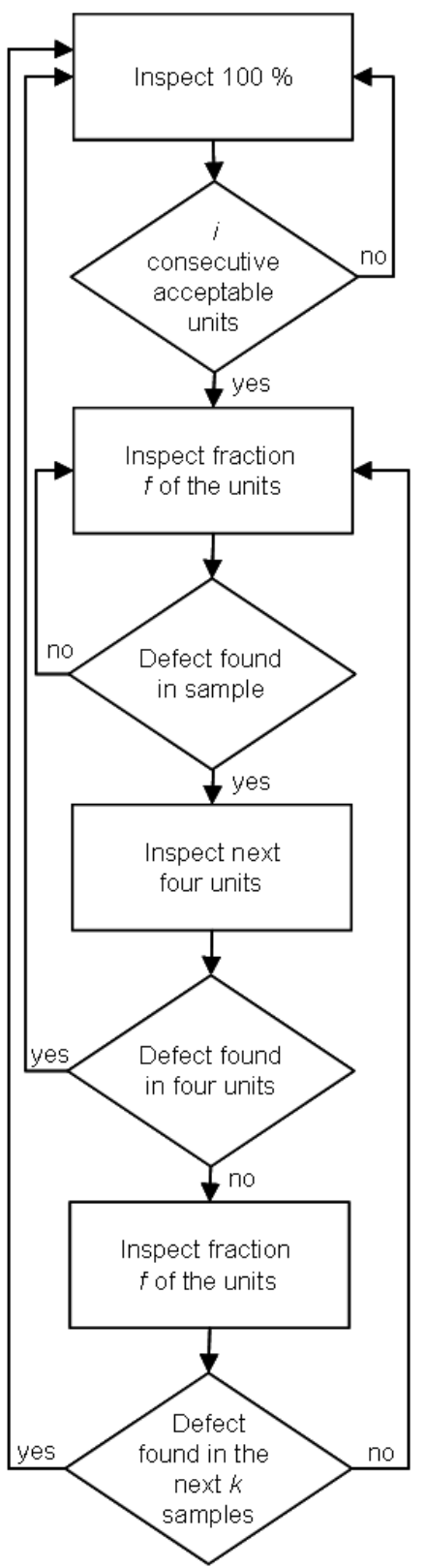

\subsection{Challenges with Conventional CSPS}

The traditional CSP methods are only effective in single test environment, and not for multiple, simultaneous tests. Modern products are complicated with numerous 
functionalities requiring testing. Relevant for the end-user is the total quality, of the complete product, rather than the performance of a single function. The shortcomings of the traditional CSPs have been tried to address, for example by Dodge (1943). Three types of approaches have been specified:

1. The chosen AOQL value applies to all tests collectively, and all the chosen tests are executed for each unit;

2. A separate AOQL is established for each test;

3. Tests are grouped into two, or more, classes with a separate AOQL assigned to match their seriousness. Tests in each class are then treated collectively.

Grouping all the tests together (option 1 in the list above), would be a simple approach to apply, but would mean that all the tests return to the $100 \%$ when any of their results trigger the return conditions. Also, when the quality target is set high (AOQL small), and the yield far from perfect, sampling cannot be started simply due to $i$ successive acceptable units not being found in practice. On the other hand, the options 2 and 3, cause administrational difficulties, when treating a large number of test classes, or a large number of separate tests (Dodge, 1943).

Also, the AOQL of every test, or a test group, adds to the cumulative AOQL value. In a multi-test environment, the cumulative AOQL determines the real worst case quality experienced by the end-user. Keeping the cumulative AOQL at a decent level requires demanding AOQL values to be set for individual tests, or test groups. Managing the $\mathrm{AOQL}$ values can be very challenging in multi-product, multi-test environments. (Stephens, 2001).

\subsection{Continuous Sampling with Multiple Tests}

Keeping in mind the drawbacks of CSP methods described above, it is obvious that some modifications should be considered. Three main targets can be identified for realisation of sampling with CSP methods in a multi-test environment:

1. Sampling can be utilised efficiently even if some of the tests fail frequently;

2. One failing test does not return all tests back to $100 \%$;

3. Only one AOQL value is used to characterise cumulative quality risk.

Considering the targets identified above, the following procedure is developed in this study, to address the challenges of sampling with multiple tests (Figure 2). 
Figure 2 Main principles of the developed modified plans

1. One common $A O Q L$ is set to cover all the tests.

2. Tests are pre-classified into two sets: those always in $100 \%$ inspection state and those potential for sampling.

3. Those identified potential for sampling are tested $100 \%$ for $i$ units: the first failing test identifying a defective product is placed into $T_{100}(100 \%)$, the rest of the test cases are placed into the sampling set $\left(T_{s}\right)$.

4. Upon failure of one of the tests in the sampling set (Ts), only the first failing test is returned to the $100 \%$ testing $\left(T_{100}\right)$.

In this procedure, a common AOQL is set to cover all the tests (Fig. 2, phase 1). This is done to reach a level of simplicity for practical environments.

All the tests are not necessarily considered for sampling (Fig. 2, phase 2), instead tests can be pre-classified on the basis of different criteria, such as, known quality flaws, criticality, or importance (see, e.g. Chen, 2005). This way, only the tests with better potential for successful sampling will be considered, alleviating the risk further. Tests that have been identified not suitable for sampling will always remain in $100 \%$.

Tests that are identified potential for sampling are tested for $i$ consecutive units, after which they enter a sampling set $\left(\mathrm{T}_{\mathrm{s}}\right)$ if no defects are found (Fig. 2, phase 3). Should a defective product be found, only the first test indicating this will be placed in $100 \%$ $\left(\mathrm{T}_{100}\right)$. In other words, any other test, following the first one, indicating the same defective product will be placed in a sampling set $\left(\mathrm{T}_{\mathrm{S}}\right)$. Overlapping of tests is common in electronics testing, and can cause additional expenses without providing any additional test coverage. This is the motivation to only act on the first test indicating a defective product. Different methods exist for analysing the overlapping of tests (see, e.g. Hird et al., 2002; Rijckaert and de Jong, 2003; Antila, 2005).

Upon a failure in sampling set $\left(\mathrm{T}_{\mathrm{S}}\right)$, the first failed test is returned to $100 \%$ testing (Fig. 2, phase 4). This is in contrast to the original procedure by Dodge (1943), where all the tests return to $100 \%$. It is only necessary to return the first failed test to $100 \%$, as multiple tests may be indicating the same defect.

A test in $100 \%\left(\mathrm{~T}_{100}\right)$ cannot be returned back to sampling $\left(\mathrm{T}_{\mathrm{S}}\right)$ without conducting all the tests for $i$ consecutive units. In other words, if there are motivations to return a failed test into the sampling set, a new clearing interval is required to all the tests that were initially identified potential for sampling.

Figure 3 presents a block diagram for modified CSP-1 procedure developed in this study, as described above. This procedure follows the main principles described above. 
Figure 3. Modified CSP-1 developed in this study

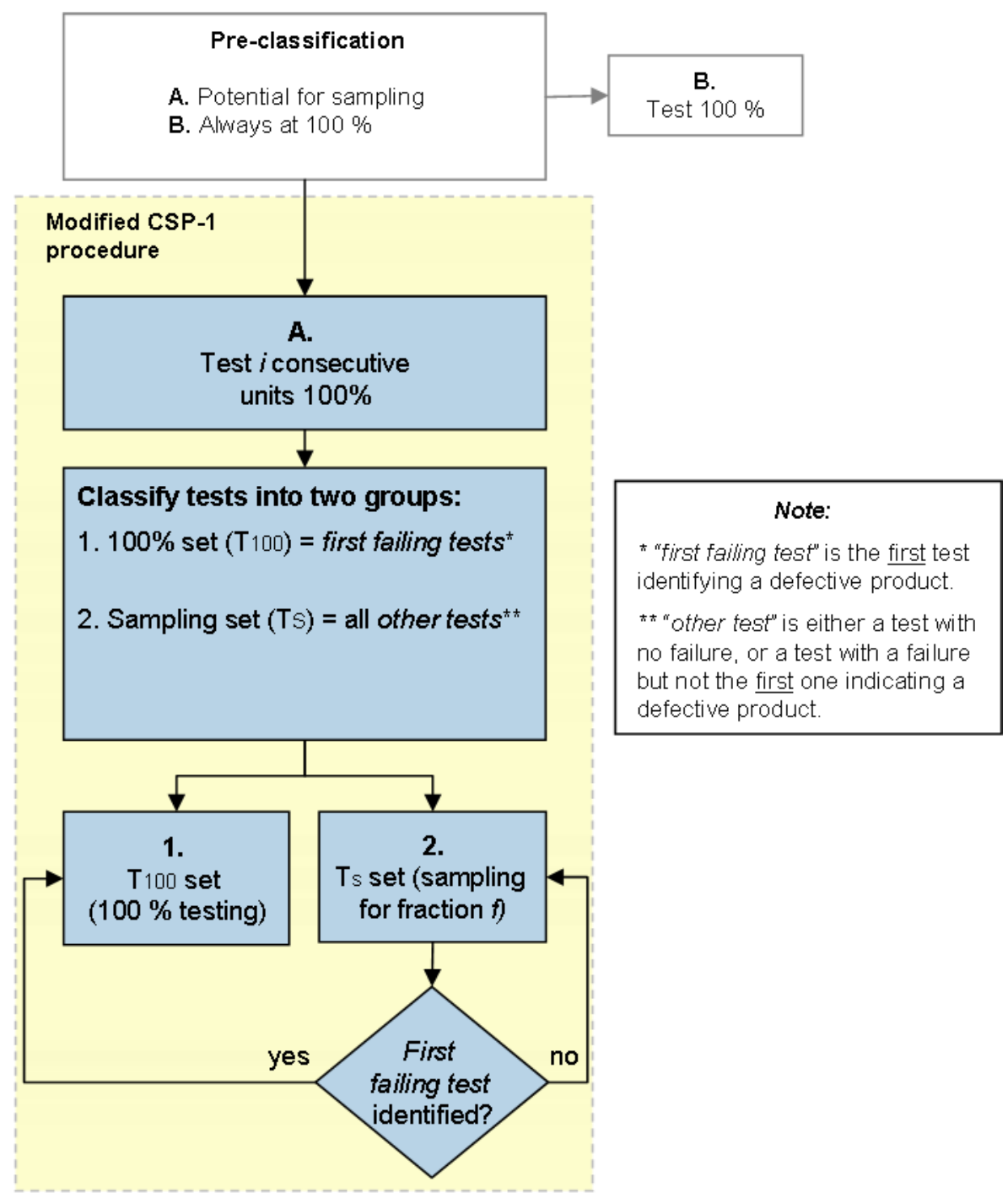

In the described modified procedure above, the AOQL can be guaranteed, due to the following reasons:

1. The AOQL of the group tested for $100 \%\left(\mathrm{~T}_{100}\right)$ is always $0 \%$;

2. The modified CSP procedure ensures that the selected AOQL is valid until one test is returned from the sampling set $\left(\mathrm{T}_{\mathrm{S}}\right)$ to $100 \%$ testing $\left(\mathrm{T}_{100}\right)$

3. After returning a test from the sample set $\left(\mathrm{T}_{\mathrm{S}}\right)$ to $100 \%$ testing $\left(\mathrm{T}_{100}\right)$, the AOQL cannot become worse than the original one.

A potential challenge of the modified procedure is the product flow being virtually infinite in length, and there would not be automated restart of the clearing interval $(i)$. The number of tests in the $100 \%$ set $\left(\mathrm{T}_{100}\right)$ would approach the total number of tests, 
initially identified potential for sampling. This will create a need to apply a clearing interval at some point, to keep the number of executed tests at a target level. In addition, a new clearing interval will be necessitated in practical terms, should the product or the process have changed (e.g. Juran, 1988). Triggering a new clearing interval must be analysed on case-by-case basis.

\subsubsection{Modified CSP-2}

The procedure for the modified CSP-2 plan is mainly the same as for the modified CSP1. Nevertheless, the modified CSP-2 allows one defect to occur without an immediate return to $100 \%$ testing. Instead, the following $k$ samples are under special observation for the test in question in the same manner as for the traditional CSP-2.

Should there be a second failure occurring during $k$ samples, the test is moved to the $100 \%$ testing. In the case of no second failure occurring during the alert period, sampling continues as prior to the first failure.

However, should a different test fail during this time, the first test triggering the alert period $k$ will be moved to $100 \%$ testing, and the test indicating a different defect will start a new alert period for the $k$ next samples.

\subsubsection{Modified CSP-3}

The modified CSP-3 adds one additional rule to the CSP-2 procedure. Any failing test triggers a period of $100 \%$ testing for the next four products, following the defective one. Should the test that caused the $100 \%$ verification period be failed again during this time, it will be moved immediately to $100 \%$ testing.

On the other hand, should there be a different failure during the next four products, the test that detected the previous failure is returned to $100 \%$ testing and a new period of four is started for the latter failing test.

Should there be no additional failures during the period of four, the sampling procedure continues with an alert period for the next $k$ samples. A sample failing during this period will cause the initiating test to be returned to $100 \%$ testing. Also, failure detected by another test to the one detecting the original defect will cause a return to 100 $\%$ testing for the initiating test, but also a new $100 \%$ testing period for the next four products. Finally, should there be no new failures being detected during the alert period, sampling continues as prior to the detection of the initiating failure.

\section{Empirical study}

\subsection{The research process}

The applicability of the modified sampling plans, developed in this study, were analysed for the purpose of real life high volume electronics production. Existing test data was utilised for the analyses. The simulations of the CSP procedures were carried out for a high volume telecommunications RF product with 2800 components. This product has 35 different test cases in the functional testing, which vary from basic DC measurements to complex RF-modulation tests. The test data included 560000 test results from 16000 manufactured products. The data was collected to obtain enough information for simulations with Dodge's original plans, and with the developed modified CSPs. The intention was to use the results for the original plans as a baseline for 
further comparisons between the different methods. For simplicity, and due to the variety among Dodge's CSP procedures, only those utilising a single AOQL for the entire test set were studied.

To be able to test the applicability of the modified plans, two experiments were performed. First, Dodge's original plans and the three developed modified plans were simulated by using a rather modest AOQL. This was done to verify the capability of meeting the set AOQL criteria, and to study the relative efficiency of the plans. The second experiment was conducted on the plan identified as the most efficient during the initial simulations. The additional simulations were performed in the same manner as the first ones, to compare the effect of different AOQL.

The empirical study utilises the concepts of, reduction ratio, stability, and escape ratio. Reduction ratio describes how sampling decreases the total number of run tests, see equation (1),

$R R=\frac{N_{100}-N_{S}}{N_{100}}$

where

$R R=$ reduction ratio

$N_{100}=$ total number of tests run in $100 \%$ testing

$N_{s}=$ total number of tests run while sampling.

The utilised sampling plan will eventually reduce the number of tests in sampling mode, depending of the found defects. By analysing the variation in the number of sampled test cases, the stability of the sampling plan is obtained. Stability, in this simulation study describes the change in the number of test cases entering sampling, and the number of test cases in sampling at the end of the simulations.

This study utilises the concept of escape ratio to describe the share of faulty products that would reach the customers as a consequence of sampling. This parameter can be obtained as the $100 \%$ test data is available for all the 16000 products, and the effect of sampling can thus be simulated. By comparing the escape ratio, and the pre-set AOQL value, the applicability of the analysed models can be confirmed.

The suitability, and efficiency, of the CSPs for high volume production can be estimated by using the parameters of reduction ratio, stability, and escape ratio. In practice, this would allow calculating the cost effect of sampling.

\subsection{Results and analyses}

\subsubsection{Experiment 1}

In the first experiment, the AOQL was set to a modest $0.53 \%$, and sampling interval $f$ was set to $1 / 10$ (see Table 1). The chosen AOQL is for simplicity, and does not represent any actual target level in production. The clearing interval $i$ and alert period $k$ were obtained as defined in (e.g. MIL-STD-1235C, 1974; Grant and Leavenworth 1996). Noteworthy is that the clearing interval $i$ is larger for the original and modified CSP-2 and CSP-3. 
Table 1. Sampling parameters for experiment $1(\mathrm{AOQL}=0.53 \% ; f=1 / 10)$.

\begin{tabular}{lccc}
\hline \multicolumn{1}{c}{ Plan } & Clearing interval $\boldsymbol{i}$ & Fraction $\boldsymbol{f}$ & Alert period $\boldsymbol{k}$ \\
\hline Dodge CSP-1 & 207 & $1 / 10$ & NA \\
Dodge CSP-2 & 275 & $1 / 10$ & 275 \\
Dodge CSP-3 & 275 & $1 / 10$ & 275 \\
Modified CSP-1 & 207 & $1 / 10$ & NA \\
Modified CSP-2 & 275 & $1 / 10$ & 275 \\
Modified CSP-3 & 275 & $1 / 10$ & 275 \\
\hline
\end{tabular}

The results for the experiment 1 are presented in Table 2. Even though the selected AOQL was fairly modest, qualification for sampling mode could not be achieved with the original CSPs. In other words, it was not possible to find 207 consecutive products with no defects among the analysed 16000 products. It would be rather unlikely for all the analysed 35 test cases to indicate $i$ successive pass results for all the tested units at the same time. The results in this environment support the view that the original CSPs are not suitable for complex high quality products. Therefore, the rest of the comparison was conducted for the modified CSP methods.

Table 2. Results of experiment $1(\mathrm{AOQL}=0.53 \%)$.

\begin{tabular}{lccccc}
\hline \multirow{2}{*}{ Plan } & Reduction & Escape & \multicolumn{3}{c}{ Stability } \\
\cline { 4 - 6 } & ratio RR & ratio & $\begin{array}{c}\text { Tests in sampling } \\
\text { at beginning }\end{array}$ & $\begin{array}{c}\text { Tests in sampling } \\
\text { at end }\end{array}$ \\
\hline Dodge CSP-1 & $0.00 \%$ & $0.00 \%$ & 0 & 0 \\
Dodge CSP-2 & $0.00 \%$ & $0.00 \%$ & 0 & 0 \\
Dodge CSP-3 & $0.00 \%$ & $0.00 \%$ & 0 & 0 \\
Modified CSP-1 & $63.18 \%$ & $0.35 \%$ & 32 & 22 \\
Modified CSP-2 & $61.69 \%$ & $0.52 \%$ & 30 & 21 \\
Modified CSP-3 & $61.52 \%$ & $0.52 \%$ & 30 & 21 \\
\hline
\end{tabular}

The simulation results for the modified methods indicate variation among the efficiency of the three modified CSPs (see Table 2). In the modified CSP-1 initially 32 tests enter sampling, and 3 test cases remain in $100 \%$ testing. In the modified CSP-2 and CSP-3, 30 tests enter sampling, and 5 remain in $100 \%$ testing. At the end of the simulation 22 test cases were in sampling mode, when using the modified CSP-1. The simulations indicate 21 test cases being still sampled at the end, while using modified CSP-2 and CSP-3. The variation among the results, the different models give, is an effect of the clearing interval, and the manner the failing tests are handled.

A longer clearing interval is an indication of fewer faults in the long run, providing stability to sampled test group $\left(\mathrm{T}_{\mathrm{S}}\right)$. In other words, the test cases that have fewer failures at the beginning of the simulation are more likely to end up being sampled also after a longer period of time. Stability of sampling decreases the need for a new clearing interval.

The results presented in Table 2 indicate the modified CSP-1 being the most effective in the simulations, in terms of reduction ratio. In addition, the number of test cases in sampling mode at the beginning, and at the end indicates the modified CSP-1 as being more efficient than the modified CSP-2 and CSP-3. 
The modified CSP-1 having the most stringent criteria for returning failed tests to 100 $\%$, makes it the most efficient in terms of escape ratio, compared to the modified CSP-2 and CSP-3 with a larger escape ratio. Nevertheless, all the studied methods are within the set AOQL.

\subsubsection{Experiment 2}

The modified CSP-1, identified as the most efficient in the simulations, was analysed for different AOQL values. This was done to obtain further understanding on how the AOQL influences the escape ratio, reduction ratio, and stability. The AOQL values of $0.53 \%, 0.12 \%$, and $0.074 \%$ were chosen for the analyses, which corresponds to clearing intervals of 207, 920 and 1470 respectively (see e.g. MIL-STD-1235C, 1974 ; Grant and Leavenworth 1996). The same fraction $f=1 / 10$ was used for sampling in all cases. The results for the experiment 2 are presented in Table 3 .

The effect of a longer clearing interval is directly visible in the results. The number of test cases in sampling at the beginning decreases with tighter AOQL. The reduction ratio became significantly worse with tighter AOQL. In other words, the total number of needed tests increases with tighter quality requirements. At the same time, the stability increases with tighter AOQL, making the sampling process more controllable.

Table 3. Results for the experiment 2.

\begin{tabular}{cccccc}
\hline & & & & \multicolumn{2}{c}{ Stability } \\
\cline { 5 - 6 } AOQL & $\begin{array}{c}\text { Clearing } \\
\text { interval } i\end{array}$ & $\begin{array}{c}\text { Reduction } \\
\text { ratio RR }\end{array}$ & Escape ratio & $\begin{array}{c}\text { Tests in } \\
\text { sampling at } \\
\text { beginning }\end{array}$ & $\begin{array}{c}\text { Tests in } \\
\text { sampling at } \\
\text { end }\end{array}$ \\
\hline $0.53 \%$ & 207 & $63.18 \%$ & $0.35 \%$ & 32 & 22 \\
$0.12 \%$ & 920 & $54.22 \%$ & $0.11 \%$ & 25 & 21 \\
$0.074 \%$ & 1470 & $49.46 \%$ & $0.06 \%$ & 23 & 20 \\
\hline
\end{tabular}

\section{Managerial implications}

Modern electronics products are very complicated causing challenges for production testing. Especially, functional testing has become a bottleneck requiring significant resources.

Sampling methods have long been used, in different fields, to make inspection activities more efficient, and to reduce costs. Sampling techniques are, however, not widely used for functional testing in telecommunications, and other modern high tech sectors. The reason for this has been the traditional methods not being directly applicable for mass-production with large number of different tests. This study also confirms this non-applicability. In order to the methods to be considered in real practical environments, they must be simultaneously applicable for complex environments, and to be simple enough. These types of solutions have not been found before.

In this study, the traditional methods have been developed further, to address the challenges of the modern electronics industry. The amount of functional testing can be reduced by utilising the developed CSP-based models. The modified CSPs will reduce the time required for testing, and consequently the costs, without sacrificing the quality level seen by the end-user. The benefit of the CSP models is the possibility to know the 
risk level, in the form of guaranteed worst case quality (AOQL). In addition, the need for expensive test equipment may be reduced, should sampling be utilised successfully.

However, managers should notice that sampling has also some drawbacks. Useful information might be lost, normally used for diagnostics and test statistics. Nevertheless, diagnostics can be supported by arranging possibility to run additional tests for faulty products and careful design of reporting systems ensuring decent statistical data also while sampling.

When properly implemented, the developed modified sampling methods can be an efficient and effective tool for rationalising functional testing, as shown by this study, where the number of tests was reduced by over fifty percent. Nevertheless, it is the duration of tests, rather than their number, that will influence the real benefit obtainable. For optimal results, sampling plans to be used and the tests to be sampled must be chosen on a case-by-case basis.

\section{Conclusions}

In modern, complex electronics production, functional testing requires a significant amount of resources, and time. Therefore, the amount of functional testing must be minimised without sacrificing the quality experienced by the end-user. Sampling is a valuable method due to the possibility of controlling the quality risks. The existing methods, however, are not directly applicable for the demanding modern environment. This study has developed new sampling procedures through both, theoretical means, and by empirically simulating real industrial test data.

This study confirms that the traditional sampling methods are not directly applicable for challenging multi-test environment. The simulations conducted demonstrate that the existing CSPs can be developed for more demanding purposes. In this study, new models were developed to better address the real industrial challenges. The developed models proved to be efficient, by allowing reducing the number of tests up to half of the original number.

When analysing the efficiency of testing in real processes, the critical parameter is the time required for conducting tests, rather than the number of these tests. The actual savings depend on the type of the product and the complexity of the tests.

The findings show that all the modified CSP procedures work in a functional board testing in continuous electronics mass-production with numerous tests. The simplest of the new plans, modified CSP-1, was found to be the most efficient based on the simulations. The more detailed descriptions and illustration of this procedure are presented in Figures 2 and 3.

This study was conducted by simulating the data for 16000 products with 35 functional tests each, resulting in total of 560000 test cases. However, studying multiple products from several manufacturers is required to confirm the wider applicability of these results. The variation in results among the three modified CSP methods was relatively small, and therefore their order of superiority may be different in other environments.

Areas worth of further study include, practical rules for forced restart of clearing intervals, and how the order of tests affect the reduction of these tests. In addition, a procedure for returning individual tests to sampling without restarting the clearing interval would be another interesting aspect. 


\section{References}

Antila, J. (2005) 'Sampling of Tests in Continuous Production', IEEE Board Test Workshop, pp. 14.

Balamurali, S., Kalyanasundaram, M. and Jun, C-H. (2005) 'Generalized CSP-(C1, C2) Sampling plan for continuous production processes', International Journal of Reliability, Quality and Safety Engineering, Vol. 12, No. 2, pp. 75-93.

Balamurali, S. and Jun, C-H. (2006) 'Average outgoing quality of CSP-C continuous sampling plan under short run production processes', Journal of Applied Statistics, Vol. 33, No. 2, pp. 139154.

Balamurali, S. and Subramani, K. (2004) 'Modified CSP-C Continuous Sampling Plan for Consumer Protection', Journal of Applied Statistics, Vol. 31, No. 4, pp. 481 - 494.

Belt, P., Harkonen, J., Mottonen, M., Kess, P. and Haapasalo, H. (2008) 'Improving the efficiency of verification and validation', International Journal of Services and Standards, Vol. 4, No.2, pp. 150 - 166.

Chen, C-H. (2005) 'Economic Design of Dodge-Romig AOQL Single Sampling Plans by Variables with the Quadratic Loss Function', Tamkang Journal of Science and Engineering, Vol. 8, No 4, pp. 313-318.

Chen, C-H. (2004) 'Minimum Average Fraction Inspected for Modified Tightened Two-level Continuous Sampling Plans', Tamkang Journal of Science and Engineering, Vol. 7, No.1, pp. $37-40$.

Chen, C-H. and Chou, C-Y. (2004) 'Minimum Average Total Inspection of Variable Lot-size Sampling Plan for Continuous Production', Journal of Applied Statistics, Vol. 31, No. 2, pp. 183-189.

Chen, C.H. and Chou, C.Y. (2002) 'A Note on the Continuous Sampling Plan CSP-V', Economic Quality Control, Vol. 17, No. 2, pp. 235-239.

Derman, C., Littauer, S. and Solomon, H. (1957) 'Tightened Multi-Level Continuous Sampling Plans', The Annals of Mathematical Statistics, Vol. 28, No. 2, pp. 395-404.

Dodge, H.F. (1943) 'A Sampling Inspection Plan for Continuous Production', The Annals of Mathematical Statistics, Vol. 14, No. 3, pp. 264-279.

Dodge, H.F. and Torrey, M.N. (1951) 'Additional continuous sampling inspection plans', Industrial Quality Control, Vol. 7, No. 5, pp. 7-12.

Gershon, M. and Christobek, M. (2006) 'Comparing the cost of quality between C $=0$ acceptance plans and MIL-STD-105E plans', International Journal of Productivity and Quality Management, Vol. 1, No.3, pp. 272 - 289.

Govindaraju, K. (2000) 'Design of generalized CSP-C continuous sampling plan', Journal of Applied Statistics, Vol. 27, No. 7, pp. 829-841.

Grant, E. and Leavenworth R. (1996) 'Statistical Quality Control', 7th edition. pp. 764.

Groomer, S.M. and Murthy, U.S. (2003) 'Monitoring High Volume On-line Transaction Processing Systems Using a Continuous Sampling Approach', International Journal of Auditing, Vol. 7, No. 1, pp. 3-19.

Guo, R-S., Chiang, D.M. and Pai, F-Y. (2007) 'A WIP-based exception-management model for integrated circuit back-end production processes', The International Journal of Advanced Manufacturing Technology, Vol. 33, No. 11-12, pp. 1263-1274.

Helo, P. (2004) 'Managing agility and productivity in the electronics industry', Industrial Management \& Data Systems, Vol. 104, No. 7, pp. 567 - 577.

Hird, K., Parker, K.P. and Follis B. (2002) 'Test Coverage: What Does It Mean When a Board Test Passes?' Proceedings of International Test Conference, IEEE, pp. 1066 - 1074

Juran, M. (1988) ‘Quality Control Handbook', 3rd edition. pp. 1774. 
Kerkhoff, H.G. (2005) 'The test search for true mixed-signal cores', Microelectronics Journal, Vol. 36, No. 12, pp. 1103-1111.

MIL-STD-1235C (1974) 'Single and multi-level continuous sampling procedures and tables for inspection by attributes'. [withdrawn standard], Department of Defense.

Lieberman, G.J. and Solomon, H. (1955) 'Multi-Level Continuous Sampling Plans', The Annals of Mathematical Statistics, Vol. 26, No. 4, pp. 686-704.

Montgomery, D. (2004) 'Introduction to Statistical Quality Control', 5th edition, pp. 776.

Rijckaert, W. and de Jong, F. (2003) 'Board Test Coverage: The Value of Prediction and How to Compare Numbers,' Proceedings of International Test Conference, Vol.2, pp. 190 - 199

Ryu, J-Y., Kim, B.C. and Sylla, I. (2006) 'A Novel RF Test Scheme Based on a DFT Method', Journal of Electronic Testing, Theory and Applications, Vol. 22, No. 3, pp. 229-237.

Stephens, K. (2001) 'The Handbook of Applied Acceptance Sampling: Plans, Principles and Procedures', pp. 538

Vasudevan, D. and Selladurai, V. (2006) 'Average outgoing quality limit and lot tolerance percent defective indexed acceptance sampling plans using artificial neural networks' International Journal of Productivity and Quality Management, Vol. 1, No.4, pp. 411 - 424. 